\title{
Emergência médica na prática odontológica no Estado de Santa Catarina: ocorrência, equipamentos e drogas, conhecimento e treinamento dos cirurgiões-dentistas
}

\author{
Medical emergency in dental practice: \\ occurrence, equipments and drugs, professional \\ expertise and training of the clinicians
}

Abstract The aim of this research was to verify the number of occurrences of medical emergencies during the odontological practice; the adequacy of equipments and drugs; the professional knowledge level and training of such professionals in dealing with medical emergencies, both in terms of public health care as well as private practice. Postal questionnaires were sent to 6.000 dentists who were member registered up to April 2004 at the Regional Dental Council of the State of Santa Catarina. There were 506 (8.43\%) responses. The analysis of the results revealed that the clinicians who replied were aware on the importance of the issue, and declared themselves not prepared to solve a medical emergency. The most frequent emergencies reported were syncope, tachycardia, hypertension, reaction to local anesthetic and hypoglycemia. The public health service has a larger supply of equipments and drugs for medical emergencies. Official letters were also sent to seven dentistry schools in the state, which answered that there are no specific credits about the subject of medical emergency. Such issue is usually briefly referred in others credits, and in the initial semesters of the course.

Key words Medical emergency, Equipments, Drugs, Public health care, Private clinics
Resumo Esta pesquisa teve por objetivo verificar a ocorrência de emergências médicas na prática odontológica, a adequação de equipamentos e drogas, e o nivel de conhecimento e treinamento dos profissionais da odontologia no enfrentamento dos eventos emergenciais, tanto no serviço público quanto na clínica privada. Os questionários foram enviados via postal, aos 6.000 cirurgiõesdentistas (CDs) inscritos até abril de 2004 no Conselho Regional de Odontologia do Estado de Santa Catarina. Houve um retorno de 506 questionários (8,43\%). A partir da análise dos dados, constatou-se que os CDs respondentes estão conscientes da importância do tema e sentem-se despreparados para solucionar uma emergência médica. As emergências relatadas com maior freqüencia foram: lipotimia, taquicardia, hipertensão, reação ao anestésico local e hipoglicemia. $O$ serviço público encontra-se com uma quantidade maior de equipamentos e drogas para emergência médica. Foi enviado, também, ofício às sete instituições de ensino superior com cursos de odontologia no Estado, consulta esta que acusou a inexistência de disciplinas específicas que tratem das emergências médicas. O tema normalmente é abordado em outras disciplinas com pequena carga horária, e nos semestres iniciais do curso.

Palavras-chave Emergência médica, Equipamentos, Drogas, Serviço público, Clínica particular 


\section{Introdução}

O cirurgião-dentista (CD) enfrenta, no cotidiano de seu trabalho, o risco de se ver face a face com eventos emergenciais, exclusivos da área médica. Tais emergências - cujo enfrentamento não pode ser evitado ou transferido, por causa dos riscos de vida e saúde a que ficam expostos os pacientes e pelo estresse emocional gerado aos profissionais - devem ser por estes assumidas de imediato.

Embora não sejam comuns, as situações de emergência médica podem ocorrer na prática odontológica de modo imprevisível, sem obedecer a regras ou padrões definidos. Para Andrade \& Ranali 1 , o aumento do número de idosos que procuram tratamento odontológico e a tendência de se prolongar a duração das sessões de atendimento podem contribuir para elevar a incidência dos episódios emergenciais. $\mathrm{O}$ aumento da expectativa de vida, segundo o autor, traz ao consultório odontológico indivíduos diabéticos, hipertensos, cardiopatas, asmáticos ou portadores de desordens renais e hepáticas, obrigando o profissional a adotar certas precauções antes de iniciar o tratamento clínico propriamente dito.

As emergências médicas podem ocorrer com qualquer indivíduo, a qualquer hora e em qualquer lugar, assim como antes, durante e após qualquer procedimento em odontologia. O cirurgião-dentista deve, portanto, estar preparado para resolvê-las. A avaliação do estado geral de saúde e a adoção de medidas preventivas aumentam a segurança clínica no atendimento de pacientes que requerem cuidados especiais (Jolly2).

Atenta-se ainda que a atuação do cirurgiãodentista é regulada pelo Código de Ética Odontológico - $\mathrm{CFO}^{3}$. Neste, seus deveres e direitos fundamentais são atribuídos para servirem de base e orientação em diversos aspectos da vida profissional. Cavali 4 afirma que os profissionais liberais da área de saúde estão submetidos às legislações do novo código civil, por ser o vigente estatuto jurídico geral das relações privadas; e ao código de defesa do consumidor, por ser o estatuto geral das relações de consumo, em razão de o paciente e o profissional liberal da área de saúde encaixarem-se, respectivamente, nos conceitos jurídicos de consumidor e fornecedor. Os cirurgiões-dentistas devem, pois, procurar uma formação profissional adequada para que os problemas de ordem ética e legal sejam minimizados na sua prática.
Desta forma, procurou-se descobrir com esta pesquisa justamente se os CDs de Santa Catarina que trabalham tanto no serviço público, quanto na clínica privada, possuem conhecimentos, treinamento e equipamentos necessários para lidar com as emergências na prática odontológica.

Buscou-se também verificar se as sete faculdades de odontologia do Estado de Santa Catarina apresentam em seus currículos disciplinas específicas que tratem das emergências médicas.

Justifica-se, portanto, a presente investigação, que se propõe alertar a comunidade odontológica em geral a observar, com mais rigor, o aprendizado e o ensino continuado sobre as emergências médicas possíveis e passíveis de ocorrerem nos consultórios odontológicos.

\section{Revisão da literatura}

As emergências médicas no consultório odontológico felizmente são raras. Segundo Peterson et al. 5 (2000), a natureza ambulatorial da prática odontológica (os pacientes gravemente debilitados geralmente estão impossibilitados de utilizar as instalações ambulatoriais) é parcialmente responsável por tal fato.

Ricci6, 7 encaminhou por via postal 4.765 questionários a cirurgiões-dentistas, estabelecidos e exercendo a profissão em 159 municípios do Estado de São Paulo, procurando avaliar técnicas, conhecimento e competência no enfrentamento das emergências médicas que pudessem ter ocorrido em suas clínicas. Os dados levantados evidenciaram um despreparo geral para os procedimentos que possibilitem acesso às vias respiratórias. Na amostra inquirida, $2 / 3$ julgavam-se capacitados para praticar massagem cardíaca e reanimação boca-a-boca. $\mathrm{O}$ autor concluiu que houve uma forte concentração de clínicos gerais (dentistas sem especialização), cerca de $75 \%$ do total, no exercício da profissão, o que aumentaria a probabilidade de eventos emergenciais, pela heterogeneidade dos atos praticados nas clínicas e consultórios. Faltaram também, segundo a pesquisa, condições técnicas e científicas que capacitassem a maioria dos cirurgiões-dentistas a atuarem nas emergências médicas, em face das deficiências curriculares dos cursos regulares, carentes de disciplinas voltadas para a semiologia, a propedêutica e a terapia médica.

Chapman ${ }^{8}$, em sua pesquisa no ano de 1995, encaminhou questionário por via postal 
para 1.250 cirurgiões-dentistas, selecionados por computador na listagem da Associação Dentária Australiana (ADA), que contava com aproximadamente 7.500 odontólogos registrados. Dos respondentes, $64 \%$ tiveram cursos de reanimação cardiopulmonar (RCP) durante a graduação, porém $77 \%$ não se sentiam competentes para realizar estas manobras (RCP). Os graduados após 1980 tinham competência em RCP no curso de graduação significativamente maior do que os formados antes de 1980 ( $\mathrm{p}<$ 0,001). Segundo o autor, ser competente em RCP e ter auxílio médico rápido são mais importantes para o dentista do que possuir e saber usar drogas emergenciais.

Atherton et al.9, 10, 11 realizaram por um período de 10 anos estudo entre dentistas da Inglaterra e Escócia, com o objetivo de avaliar a prevalência, natureza e desfecho das emergências médicas em consultório odontológico. Foram enviados questionários por via postal a 1.000 dentistas da Inglaterra//País de Gales, e 500 aos profissionais da Escócia. Os autores demonstraram, nesta pesquisa, que o evento ocorreu mais comumente a cada 3,6 e 4,5 anos de prática clínica. Isso sugere que o cirurgião-dentista vivenciará entre 9 e 11 emergências médicas, em 40 anos de profissão. Curiosamente, contrariando alguns autores, o estudo revelou que a maioria dos eventos ocorreu durante procedimentos conservadores (52,2\%) não-cirúrgicos, provavelmente, pelo maior tempo gasto nesses procedimentos. Nenhuma das mortes reportadas nesse estudo ocorreram após anestesia geral.

Já num estudo realizado por Gindler et al.12, por meio de questionário via postal para 887 cirurgiões-dentistas britânicos, observouse que o evento de maior ocorrência nos seus consultórios foi a síncope vasovagal. Somente 20,8\% dos dentistas sentiam-se competentes para diagnosticar a causa das emergências nas cirurgias dentais. Os autores concluíram com tal pesquisa que, após a graduação, deveriam ser realizados treinamentos periódicos em emergência médica em consultório odontológico, para melhorar o conhecimento e a habilidade dos cirurgiões-dentistas e diminuir os casos de óbitos.

Broadbent $\&$ Thomson 13 realizaram uma pesquisa com 314 cirurgiões-dentistas da Nova Zelândia e encontraram uma prevalência de $65,2 \%$ de emergências médicas relatadas em um período de 10 anos, ou seja, uma média de 2 eventos para 10.000 em pacientes tratados com anestesia local, ou outras formas de con- trole da dor ou sedação. De todos os respondentes, 20\% sentiram-se inadequadamente preparados para resolver uma emergência médica, e a maioria sentia-se receptiva à idéia de receber futuros treinamentos. Mais da metade dos dentistas pesquisados estavam insatisfeitos com o treinamento recebido na universidade. Um em 20 CDs não sentia necessidade de receber treinamento futuro.

Alves et al. 14 aplicaram um questionário a um grupo amostral de 50 cirurgiões-dentistas na cidade de Belo Horizonte, considerando, na apuração dos resultados, o ano de formatura, a instituição e a especialidade do profissional. Os pesquisadores elaboraram um questionário com o objetivo de verificar a habilidade do cirurgião-dentista na realização da RCP, como e onde o profissional teve acesso às informações a respeito da técnica e quais os equipamentos disponíveis em seu consultório. Os autores concluíram que a experiência clínica dos profissionais da área odontológica em emergência médica não era muito grande, talvez pelo fato de a importância do conhecimento deste assunto não ter sido enfatizada nos cursos de graduação de odontologia.

Também Marzola \& Griza15 realizaram uma pesquisa com 271 profissionais e acadêmicos de odontologia, durante a VI Jornada Odontológica do oeste do Paraná. Essa pesquisa constava de sete gravuras representando as etapas das manobras de ressuscitação cardiorrespiratória (RCP) removidas de um livro-texto, montadas de forma aleatória/desorganizada. Os pesquisados deveriam ordenar as figuras de acordo com a seqüência correta destas manobras. $\mathrm{O}$ percentual de respostas erradas no grupo testado foi elevado, mostrando que profissionais e acadêmicos de odontologia não estavam capacitados a realizar com segurança o protocolo de ressuscitação cardiorrespiratória. Segundo os autores, havia necessidade de um contínuo treinamento a respeito deste assunto.

\section{Metodologia}

Esta pesquisa foi realizada por meio de questionário enviado via postal para todos os 6.000 cirurgiões-dentistas inscritos no Conselho Regional de Odontologia de Santa Catarina (CROSC), em atividade, até abril de 2004 (segundo dados do CRO-SC).

O questionário confidencial, anônimo e formatado para leitura óptica, foi elaborado 
com perguntas "fechadas", e respostas do tipo sim/não ou múltipla escolha. Foi solicitado que os respondentes devolvessem o questionário (que tinha postagem pré-paga, carta-resposta) no prazo máximo de cinco dias. Também foi enviado um ofício aos coordenadores das sete instituições de Ensino Superior do Estado de Santa Catarina que oferecem cursos de graduação em odontologia, para verificar a existência de disciplinas específicas que tratem da questão das Emergências Médicas, ou de disciplinas gerais em que este tema seja abordado.

\section{Resultados}

Dos 6.000 questionários enviados aos cirurgiões-dentistas do Estado de Santa Catarina, $506(8,43 \%)$ retornaram por via postal. Os questionários foram devolvidos por odontólogos de 105 cidades do Estado. Foi possível obter este dado por meio do carimbo do correio na carta-resposta identificando a cidade remetente.

Como houve questões que poderiam ou não ser respondidas pelos entrevistados, e como a leitura óptica anula respostas duplas, foram consideradas para cálculo dos resultados e para a confecção dos gráficos e tabelas somente as respostas válidas, desprezando-se as respostas anuladas ou em branco.

Após análise dos resultados por meio de leitura óptica, foi utilizado o programa de entrada e processamento do banco de dados StatsDirect versão 2.35, a partir da montagem de estruturas. Com base no questionário aplicado, obteve-se o seguinte resultado: 76,9\% dos cirurgiões-dentistas não se sentem preparados para solucionar uma emergência no consultório odontológico (Gráfico 1).

De acordo ainda com o estudo realizado, $88,7 \%$ dos pesquisados declararam que seus auxiliares (THD - técnico em higiene dental ou ACD - auxiliar de consultório dentário) não possuíam treinamento em emergência médica no consultório odontológico (Gráfico 2).

Os resultados mostraram também que dos $22,5 \%$ dos respondentes que trabalham com uma equipe de apoio (médico ou enfermeiro), $78,7 \%$ sentem-se mais seguros no enfrentamento de uma emergência médica (Gráfico 3).

A pesquisa revelou ainda quais as principais emergências médicas vivenciadas pelos cirurgiões-dentistas nos últimos 12 meses. Os resultados podem ser analisados no gráfico 4 .

\section{Gráfico 1}

Distribuição de freqüência da variável, sensação de estar preparados para atender emergências médicas.

\section{Gráfico 2}

Distribuição de freqüência da variável, auxiliares possuem algum treinamento em emergência médica.

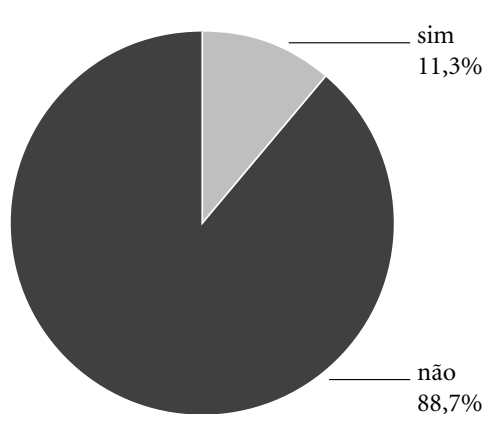

\section{Gráfico 3}

Distribuição de freqüência, da variável, sensação de segurança com equipe de apoio, entre os entrevistados da pesquisa que responderam SIM, para equipe de apoio.

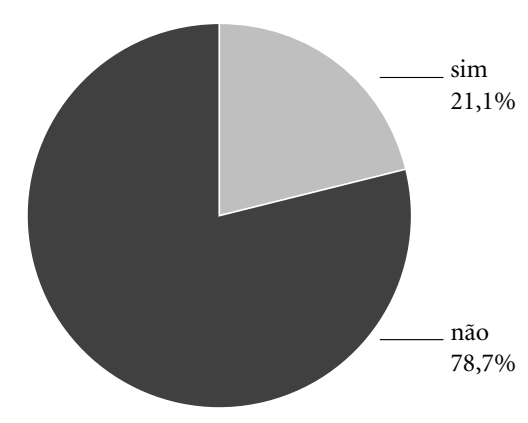




\section{Gráfico 4}

Distribuição de freqüência da variável, emergências médicas vivenciadas pelos pacientes no consultório nos últimos 12 meses, dos entrevistados da pesquisa.

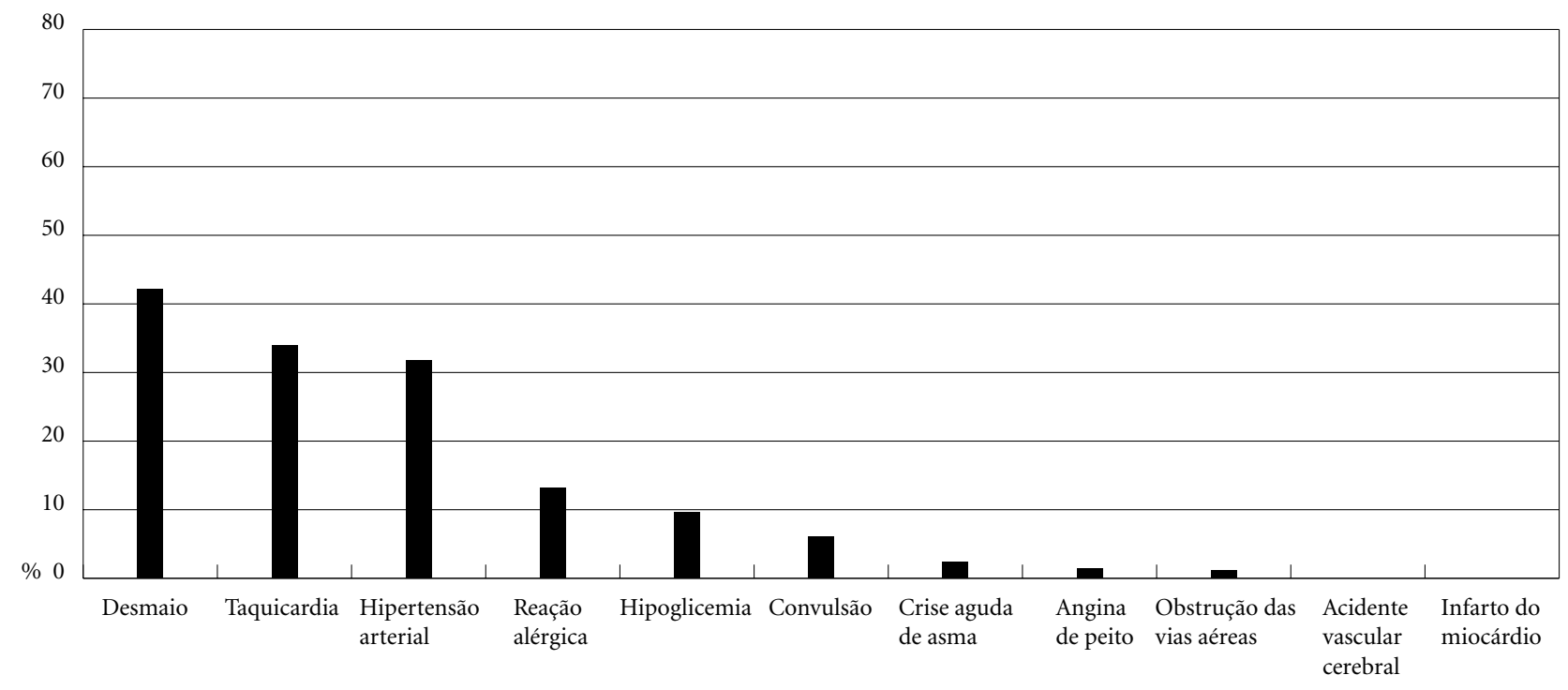

Os resultados da pesquisa, a freqüência de emergências médicas vivenciadas pelos pacientes no consultório odontológico dos entrevistados, nos últimos 12 meses são mostradas no gráfico 5 .

O gráfico 6 mostra de quais equipamentos os cirurgiões-dentistas dispõem com maior freqüência em seu local de trabalho (serviço público ou clínica particular) para solucionar uma emergência médica.

\section{Conclusão}

Neste estudo, verificou-se que mais da metade dos CDs respondentes ao questionário enviado $(76,9 \%)$ sentem-se despreparados diante de uma emergência médica em seus consultórios, independentemente de trabalhar serviço público ou clínica privada.

Apesar de 45,8\% dos respondentes terem recebido treinamento e informações sobre emergência médica em odontologia na universidade e $37,5 \%$ em cursos de pós-graduação, $16,6 \%$ nunca tiveram qualquer treinamento ou cursos nesta área. Estes dados revelam que a grande maioria dos CDs pesquisada recebeu treinamento ou informação sobre as emergências

\section{Gráfico 5}

Distribuição de freqüência da variável, freqüência de emergências médicas vivenciadas pelos pacientes no consultório nos últimos 12 meses.

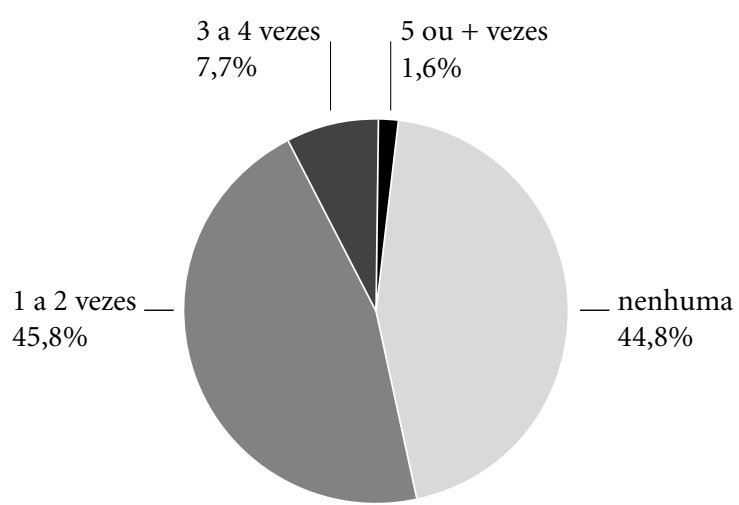


Gráfico 6

Distribuição de freqüência da variável, drogas e medicamentos disponíveis para emergências médicas no local de trabalho, tanto em consultórios particulares como em serviços públicos, dos respondentes a esta questão.

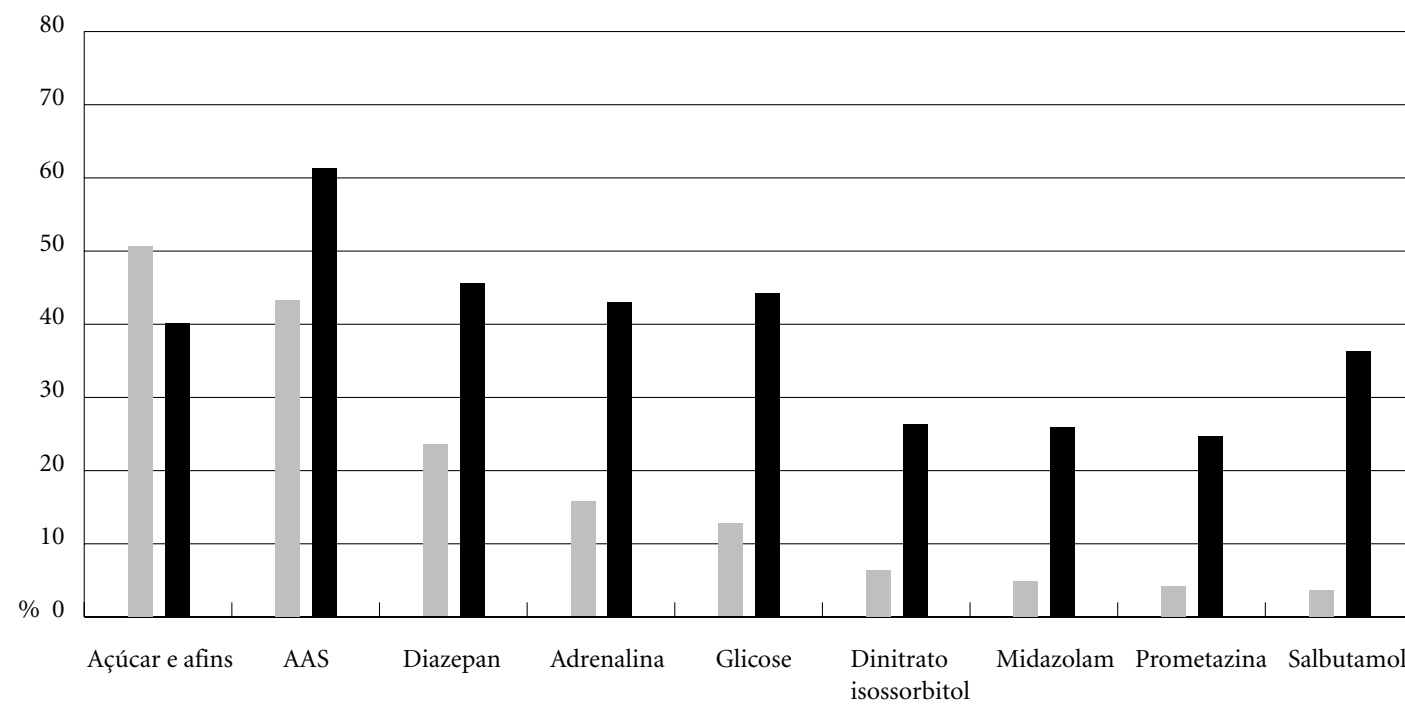

Clínica particular

médicas. Como não há, porém, uma obrigatoriedade de cursos regulares de capacitação, o assunto torna-se esquecido por não ser colocado em prática periodicamente, já que as emergências médicas são consideradas situações raras no consultório odontológico.

Verificou-se na pesquisa que $88,7 \%$ do pessoal auxiliar (ACD ou THD) que trabalha junto com os cirurgiões-dentistas respondentes não recebeu qualquer treinamento em emergência médica. Deve-se destacar a importância do treinamento e da capacitação dos auxiliares, para que os membros da equipe de atendimento odontológico possam ser capazes de reconhecer e ajudar a solucionar qualquer emergência médica.

Os dados da pesquisa mostram que $78,7 \%$ dos respondentes sentem-se mais seguros ao trabalharem com uma equipe multidisciplinar de apoio. Na maioria dos casos relatados, esta equipe é formada por médico e enfermeiro $(68,9 \%)$. Este dado revela que o trabalho pode ser mais tranqüilo para o cirurgião-dentista, quando ele atua com outros profissionais da área da saúde, diminuindo, desta forma, a insegurança, a impotência que a consciência do despreparo traz ao profissional e o estresse permanente que o acompanha e o desgasta psicologicamente.
As principais emergências médicas na prática odontológica relatadas pelos respondentes foram: síncope $(42,1 \%)$, taquicardia $(33,9 \%)$, hipertensão $(31,8 \%)$, reação alérgica ao anestésico local $(13,2 \%)$, hipoglicemia $(9,7 \%)$ e convulsão $(6,1 \%)$. Os níveis percentuais de emergências graves (eventos complexos) relatadas são preocupantes e envolvem sérios riscos de vida e saúde - para os pacientes - e ético-legais - para os profissionais. Merecem, assim, atenção especial.

Dentre todos os pesquisados houve a declaração de um caso de óbito em consultório odontológico. Conclui-se, desta forma, que são raros na prática odontológica os casos de óbito relacionados com as emergências médicas.

As providências tomadas pelos CDs pesquisados, ante as emergências médicas enfrentadas, levaram a grande maioria $(92,9 \%)$ a atender seus pacientes que vivenciaram estas emergências no próprio consultório, sem a necessidade ou a possibilidade do encaminhamento ao médico. Mais de $40 \%$ dos respondentes declararam de 1 a 2 emergências médicas vivenciadas por seus pacientes nos últimos 12 meses.

Com referência à capacidade instalada em equipamentos para emergência médica nas áreas de atuação, concluiu-se que há insuficiência de recursos materiais e equipamentos 
adequados ao atendimento básico dos eventos emergenciais que possam ocorrer na prática odontológica. Os principais equipamentos citados pelos respondentes foram: esfigmomanômetro, estetoscópio, seringas e agulhas descartáveis, suctor de saliva, saco de papel, oxigênio e AMBU. Verificou-se um maior número de equipamentos no serviço público, com exceção do suctor de saliva (bomba a vácuo), um equipamento caro e substituído por sugadores que são acoplados nos equipamentos odontológicos convencionais.

Com relação aos medicamentos, mais da metade dos pesquisados afirmam ter em seus consultórios balas e sucos que poderiam ser utilizados para os casos de hipoglicemia. As principais drogas indicadas para emergência médica citadas pelos respondentes foram: Ácido Acetil Salicílico, Diazepan, Adrenalina e Glicose. Da mesma forma que os equipamentos, a quantidade de drogas encontradas no serviço público é maior que na clínica privada, já que normalmente o atendimento médico e odon- tológico é realizado no mesmo local, havendo maior possibilidade e disponibilidade de medicamentos.

Os cursos de odontologia do Estado de Santa Catarina não apresentam em seus currículos disciplinas específicas que tratem da importante temática das emergências médicas na prática odontológica. $\mathrm{Na}$ grande maioria das universidades, o tema é abordado em outras disciplinas, com pequena carga horária e nas primeiras fases do curso. As principais disciplinas em que o tema é tratado são: anestesiologia, cirurgia ou primeiros socorros. Com base nos dados encontrados na pesquisa, destaca-se a necessidade de uma reformulação dos currículos escolares, para melhor preparo dos profissionais da odontologia, visando integrá-los à medicina básica e de rotina. Da mesma forma, é de suma importância a criação de cursos obrigatórios após a graduação, regulamentados pelo CRO e realizados periodicamente com aulas práticas e teóricas, para todos os cirurgiõesdentistas do Estado de Santa Catarina.

\section{Colaboradores}

JC Santos trabalhou na concepção teórica, elaboração, redação final do texto, organização e execução da pesquisa. D Rumel participou como orientador durante todas as etapas da elaboração da dissertação e do artigo.

\section{Referências}

1. Andrade ED, Ranali J. Emergências médicas em odontologia. São Paulo: Artes Médicas; 2002.

2. Joly DE. Evaluation of the medical history. Anesth Prog 1995; 42:84-9.

3. Conselho Federal de Odontologia. Código de Ética Odontológica. Rio de Janeiro: CFO; 2003.

4. Cavali RS 2003. A responsabilidade civil do profissional liberal da área da saúde no direito brasileiro. Revista ABO 2003; 7(4):5-6.

5. Peterson LJ, Edward E III, James RH, Myron RT. Cirurgia oral e maxilo-facial contemporânea. Rio de Janeiro: Koogan; 2000.

6. Ricci A, Bijela VT, Moraes N. O cirurgião-dentista face às emergências médicas. Parte I - Avaliação da capacidade profissional. Rev Paulista Odontol 1989; 3(11):18-35

7. Ricci A, Bijela VT, Moraes N. O cirurgião-dentista face às emergências médicas. Parte II - Avaliação dos recursos materiais disponíveis. Rev Paulista Odontol 1989; 4(11):46-51

8. Chapman PJ 1997. Medical emergencies in dental practice and choice at emergency drugs and equipment: survey of Australian Dentists. Aust Dent J 1997; 2(42):103-8.

9. Atherton GJ, McCaul JA, Wilians SA. Medical emergencies in general dental practice in Great Britain Part 1: Their prevalence over 10 year period. Br Dent J 1999; 186(2):72-9.

10. Atherton GJ, McCaul JA, Wilians SA. Medical emergencies in general dental practice in Great Britain Part 2: Drugs and equipment possessed by GPDs and used in the management of emergencies. Br Dent $J$ 1999; 186(3):125-30.

11. Atherton GJ, McCaul JA, Wilians SA. Medical emer- 
gencies in general dental practice in Great Britain Part 3: Perceptions of training and competence of GDPs in their management. Br Dent J 1999; 186(5): 234-37.

12. Gindler NM, Smith DG. Prevalence of emergency events in British dental practice and emergency management skills of British Dentists. Resuscitation 1999; 2(41):159-67.

13. Broadbent JM,Thomson WM 2001. The readiness of New Zealand general dental practitioners for medical emergencies. N Z Dent J 2001; 429(97):82-6.
14. Alves LCF, Noman-Ferreira LC, Peroni LD, Santoro LC, Lima TKS, Lopes E et al. Reanimação cardiopulmonar. Avaliação de cirurgiões-dentistas de Belo Horizonte. Jornal de Assessoria ao Odontologista 2001; 28(4):27-32.

15. Marzola C, Griza GL. Profissionais e acadêmicos de odontologia estão aptos para salvar vidas. Jornal de Assessoria ao Odontologista 2001; 27(4):19-27.

16. Conselho Regional de Odontologia. [acessado em 10 mar 2003 e 4 abr 2004]. Disponível em: www.crosc. org.br.

Artigo apresentado em 11/04/05

Aprovado em 20/10/05

Versão final apresentada em 17/11/05 